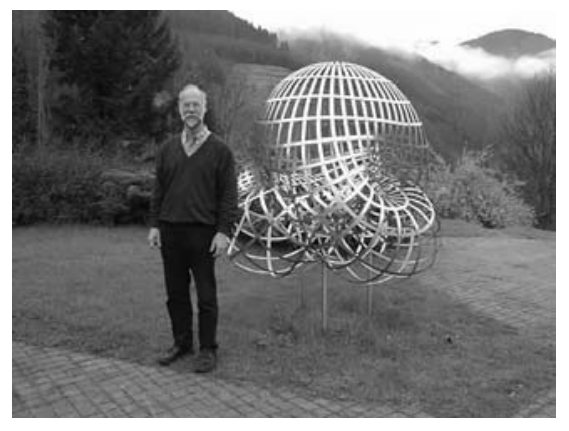

Prof. Matthias Kreck vor der Boyschen Fläche, Oberwolfach

\title{
Wird die deutsche Forschungsförderung der Mathematik gerecht?
}

\section{von Matthias Kreck}

Der nachfolgende Text ist im wesentlichen identisch mit einem Vortrag, den ich im Mai 1999 beim Bayerischen Mathematischen Kolloquium gehalten habe. Die angegebenen Zahlen sind nicht mehr aktuell (sie beziehen sich auf das Jahr 1998). Meine grundsätzlichen Überlegungen würden heute trotz gewisser Veränderungen bei der DFG nicht anders aussehen.

Meine Ausführungen sind ein Diskussionsbeitrag. Ich nehme nicht in Anspruch, zu dem Thema ernsthaft gearbeitet $\mathrm{zu}$ haben und deshalb fundierte Kenntnisse, Argumente und Thesen vorlegen zu können. Allerdings bin ich in den letzten Jahren wegen meiner Tätigkeit für das Oberwolfacher Institut und als einer der sechs gewählten Fachgutachter für Mathematik der DFG intensiver mit verschiedenen Instrumenten der Forschungsförderung in Kontakt gekommen, und so ist zum einen ein Stück Erfahrung entstanden, und es haben sich zum anderen einige Gedanken und Überlegungen entwickelt, über die ich im Folgenden berichten möchte.

Ich möchte mit einer Zusammenstellung der verschiedenen Instrumente der außeruniversitären Forschungsförderung beginnen. Ich beginne mit den Programmen, die von der DFG finanziert werden, weil sie sowohl vom finanziellen Umfang mit etwa 45 Mio. DM wie von der wissenschaftlichen Bedeutung her im Zentrum stehen.

Für mich steht unter den Fördermöglichkeiten der DFG das Normalverfahren an erster Stelle. Antragsberechtigt sind alle promovierten Mathematiker. Wie bei der DFG üblich werden Projekte gefördert. Der Antragsteller muss also ein bestimmtes Forschungsprojekt formulieren und kann für dieses finanzielle Mittel beantragen. Diese können bei PostDocs ad personam bestimmt sein, in der Regel im Rahmen eines Stipendiums, wobei ein Ortswechsel, speziell ins Ausland, besonders gern gesehen wird. Häufig zielen diese Stipendien auf eine Habilitation. Bei bereits durch eine Stelle abgesicherten Kollegen werden die finanziellen Mittel in der Regel für die Einstellung eines Mitarbeiters - das können ein oder mehrere Doktoranden sein oder auch ein PostDoc - beantragt. Aber auch Gästemittel können beantragt werden. Dies war früher in der DFG unüblich und bis vor kurzem ein Privileg der Mathematik. Inzwischen können Gästemittel von allen Fächern beantragt werden. Natürlich können auch Mittel für eigene Reisen zur Durchführung des Projektes beantragt werden. Auch Tagungen und Workshops können mit Mitteln der DFG finanziert werden.

Der Umfang dieser im Normalverfahren vergebenen DFG-Mittel betrug im Jahre 1998 etwa 12 Mio. DM.

Dies sind aber nicht die einzigen Vorhaben, die im Normalverfahren finanziert werden. Ein beachtlicher Teil dieses Kuchens geht in die Forschergruppen. Hier handelt es sich um Gruppen von etwa 6 Kollegen, die zu einem nicht zu eng gefassten, besonders attraktiven Thema, zusammenarbeiten wollen. Es gibt zur Zeit vier Forschergruppen. In etwa bekommen diese Gruppen je 1 Mio. DM. Von diesem Geld können Mitarbeiter, Gäste und - in geringem Umfang - für die Forschung unverzichtbare Geräte finanziert werden. Der Anteil der Forschergruppen am Normalverfahren ist mit etwa 4 Mio. DM recht beachtlich.

Eine ähnliche Funktion - nur umfangreicher, breiter und interdisziplinärer - wie die Forschergruppen haben die Sonderforschungsbereiche. Zur Zeit gibt es 8 mathematische Sonderforschungsbereiche. Der Umfang beträgt pro SFB etwa 2 Mio. DM.

Während Forschergruppen und SFBs in der Regel zumindest schwerpunktmäßig an eine Hochschule ange- 
gliedert sind, zielt die nächste Stufe der Förderung darauf ab, ein besonders attraktives Forschungsgebiet national zu fördern durch die Schwerpunktprogramme. Hier tritt eine Gruppe von z. B. sechs Kollegen aus verschiedenen Universitäten als Antragsteller auf. Mit der Bewilligung eines solchen Antrages ist nur ein Rahmen genehmigt, der es jedem fachlich passenden und qualifizierten Kollegen aus Deutschland erlaubt, hinterher einen Antrag auf Finanzierung eines Doktoranden oder PostDoc zu stellen. In der Mathematik gab es bis vor kurzem nur vier solche Schwerpunktprogramme. Letzte Woche wurden zwei weitere Anträge bewilligt, ein sehr großer und ungewöhnlicher Erfolg für die Mathematik. Der Umfang dieses Programmes für die Mathematik beträgt gut 10 Mio. DM. Es sei noch ergänzend darauf hingewiesen, dass ein mathematiknaher Antrag, nämlich zur String Theorie, ebenfalls erfolgreich war.

Als nächstes möchte ich an die Graduiertenkollegs erinnern, von denen es in der Mathematik immerhin 23 gibt. Je nach Größe werden je etwa sechs bis zehn Doktoranden und ein oder zwei PostDocs finanziert. Ich schätze den finanziellen Umfang pro Kolleg etwa auf 500.000, den Gesamtumfang also auf 12 Mio. DM.

Zum Schluss sei noch auf Programme zur Einzelförderung von Wissenschaftlern hingewiesen, wobei mir das Heisenberg-Programm für habilitierte Spitzenkandidaten, bei denen man davon aus geht, dass sie sehr gute Chancen bei einer Berufung auf eine Professur haben, besonders wichtig erscheint. Dann gibt es den Hess-Preis, der einem jungen Wissenschaftler den Aufbau einer kleinen Forschergruppe ermöglicht, sowie seit neuestem das Emmy-Noether-Programm. Schließlich gibt es noch die „S-Klasse“ in der DFG, die Leibniz-Preise, die besonders hervorragenden Wissenschaftlern etwa 1,5 Mio. DM Forschungsgelder zur Verfügung stellen.

Alles in allem gibt es in der DFG ein vielseitiges und finanziell umfangreiches Instrumentarium zur Forschungsförderung. Wenn ich umfangreich sage, so ist dies noch genauer zu diskutieren. Verglichen mit den Gesamtausgaben der DFG sind die 45 Mio. DM, die in die Mathematik gehen, nämlich eher gering, es handelt sich um $2 \%$ der Fördermittel der DFG.

Ich will nun kurz auf Forschungsförderung der Mathematik außerhalb der DFG eingehen. Hier denke ich in erster Linie an die beiden Max-Planck-Institute in Bonn und Leipzig, bei denen jeweils vier Direktoren auf Dauer beschäftigt sind, und wo ein umfangreiches Gästeprogramm von Mathematikern, die für typischerweise bis zu ein Jahr lang am Institut arbeiten, durchgeführt wird. Daneben gibt es das Mathematische Forschungsinstitut Oberwolfach, dessen Funktion allgemein bekannt sein dürfte. Schließlich gibt es spezifische Institute wie das Weierstraß-Institut und das Zuse-Zentrum in Berlin oder das Institut für diskrete Mathematik in Bonn, die einen starken Anwendungsbezug haben.

Ich möchte mit meinen Äußerungen provozieren und spitze deshalb zu auf Kosten von Nachdenklichkeit, die zumeist im Hintergrund vorhanden ist. Ich möchte mit einem Punkt beginnen, der mir ziemlich wichtig ist. Ich habe 1966 angefangen zu studieren und hatte das Glück, zufällig in Bonn an dem Ort zu sein, der in der mathematischen Forschung besonders stark ist. Ich habe mich dort bis auf eine kurze Unterbrechung von einem Jahr bis 1976 aufgehalten, als ich einen Ruf nach nach Wuppertal annahm. Ich kann mich nicht erinnern, in diesen 10 Jahren das Wort Projekt auch nur einmal gehört zu haben. Man arbeitete an Problemen. Welche Probleme man aufgriff - und aufgreifen ist das richtige Wort - war wesentlich vom mathematischen Umfeld und vom Prozess der Entwicklung bestimmt. Der Zufall spielte eine große Rolle, die Kommunikation mit möglichst starken anderen Mathematikern ebenfalls. Ich habe 1972 mit einer ziemlich schwachen Dissertation promoviert und dann drei Jahre Theologie studiert, ohne ernsthaft über mathematische Forschung nachzudenken. Im Jahre 1975 habe ich mir ein Problem vorgenommen, das ich sehr interessant fand und wo ich eine naive Lösungsidee hatte. Innerhalb weniger Wochen habe ich das Problem gelöst und erst danach gehört, dass sich erfolgreiche Mathematiker wie Thom, Browder und Sullivan vorher um eine Lösung bemüht hatten, zum Glück erfolglos.

Ich schildere das so genau, weil ich mir ziemlich sicher bin, dass ich, wenn ich mich nach der Promotion um eine Förderung im Rahmen der DFG hätte bemühen müssen, mir dieses Problem nicht vorgenommen hätte. Ich hätte nämlich einen Projektantrag schreiben müssen. Dazu hätte ich, wenn ich überhaupt auf das oben angesprochene Problem gekommen wäre, die Literatur sammeln müssen, wäre auf die Versuche der berühmten Leute gestoßen und hätte meine Erfolgschancen als zu gering eingeschätzt. Außerdem wäre ich davon ausgegangen, dass die Gutachter angesichts der Schwere des Problems die Augenbrauen hochgezogen hätten, weil die Erfolgsaussichten sehr gering waren. Ich hätte also, um erfolgreich zu sein, eine gute Mischung aus einem interessanten und realistischen Problem finden müssen.

Nicht nur aufgrund dieser eigenen Erfahrung, sondern vielmehr, weil ich glaube, dass Flexibilität eine der wichtigsten Voraussetzungen für erfolgreiche Forschung ist, ist mein erster Kritikpunkt: Das Denken in Projekten ist für die Mathematik nicht angemessen.

Nun komme ich zu einem weiteren Kritikpunkt. Dass wir bei Anträgen an die DFG gezwungen sind, Pro- 
jekte zu formulieren, hat damit zu tun, dass die DFG, soweit ich das erkennen kann, alle Programme fächerübergreifend konzipiert. Es gibt sicher eine Reihe guter Gründe, die für dieses Prinzip sprechen. Aber ich bin davon überzeugt, dass es stärkere Gründe dafür gibt, dieses Prinzip aufzugeben. Es liegt auf der Hand, dass sich die verschiedenen Programme in erster Linie an den Fächern orientieren, die zur Durchführung von mit Mitteln einer Universität nicht zu finanzierenden Forschungsaktivitäten auf große Mittel angewiesen sind. Dass es für experimentelle Fächer solche Möglichkeiten geben muss, überzeugt mich sofort. Dass solche Aktivitäten schon aus logistischen Gründen geplant werden müssen, liegt auf der Hand. Dass man dafür Instrumente wie Sonderforschungsbereiche geschaffen hat, ist zu begrüßen und ein Segen für die Wissenschaft. Dass man diese Instrumente zur allgemeinen Norm erhebt, überzeugt mich dagegen überhaupt nicht. Ich erkenne an, dass die DFG sich der Problematik natürlich bewusst ist und eine flexible Durchführung erlaubt, die häufig sogar die Richtlinien sprengt. Aber trotzdem bleibt bei mir ein ungutes Gefühl. Warum kann eine Organisation, in der Wissenschaftler das Sagen haben, nicht das tun, was für Wissenschaftler sonst unverzichtbar ist, nämlich bei den Programmen differenzieren. Ich fasse meinen zweiten Kritikpunkt so zusammen: Das Prinzip, alle Programme fächerübergreifend zu gestalten, ist nicht adäquat.

Nun könnte man einwenden, dass diese Kritik an der Wirklichkeit vorbeigeht, weil - wie oben erwähnt die DFG in der Praxis so flexibel ist, dass die einzelnen Fächer ihren Eigenheiten entsprechend doch angemessen gefördert werden können. Um diesen Einwand zu diskutieren, müsste man auf die einzelnen Programme genau eingehen. Ich will das an dieser Stelle nicht tun, sondern einen radikalen Schritt machen, indem ich meine Vision vorstelle, wie eine adäquate Forschungsförderung der Mathematik aussehen sollte.

\section{Vorschläge für eine verbesserte Forschungsförderung der Mathematik}

Ich möchte diese Vorschläge an der Wirklichkeit und den Bedürfnissen des mathematischen Lebens, so wie ich es sehe, orientieren. Nach wie vor ist ein Großteil der mathematischen Arbeit ein sehr individueller Vorgang. Mit individuell meine ich nicht, dass nur Einzelpersonen agieren, sondern in erster Linie, dass der oder die (zwei, drei, manchmal auch vier) Forscher sämtliche Arbeitsschritte selbst durchführen und nicht an andere delegieren. Dies scheint mir ein großer Unterschied zu den experimentellen Fächern zu sein. Für die Durchführung der Arbeit benötigt der Forscher in erster Linie Zeit und in zweiter Linie Zugang zu Informationen, insbesondere Bibliotheken, sowie den Kontakt mit Fachkollegen, um Ideen auszutauschen und Anregungen zu erhalten. Den Zugang zu Bibliotheken braucht er ständig, den Kontakt zu Fachkollegen am liebsten natürlich auch. Das Letztere ist unrealistisch, weil je nach Entwicklung der Forschung sehr verschiedene Diskussionspartner notwendig sind. Anders sieht die Sache natürlich aus, wenn man gemeinsam an einem Problem arbeitet. Wenn sich das ergibt, so muss es Möglichkeiten zu kürzeren oder längeren gemeinsamen Aufenthalten geben.

Als wichtige dritte Komponente einer langfristigen Forschungsarbeit möchte ich die Möglichkeit nennen, Diplomanden und besonders Doktoranden einzubinden. Dies dient in erster Linie der Weiterentwicklung des Gebietes und in zweiter Linie ebenfalls dem Austausch.

Vor diesem Hintergrund entwickle ich nun meine Vorstellung von Forschungsförderung. Zentraler Baustein wäre dabei eine großer Topf, aus dem grants finanziert werden können. Diese sollten den in der Forschung durch besondere Leistungen Hervorgetretenen die Möglichkeit bieten

(a) Gäste für kürzere Zeiträume von einem Tag für einen Vortrag bis zu ein paar Wochen einzuladen,

(b) eigene Reisen zum Besuch besonders attraktiver Kollegen oder Tagungen zu finanzieren.

Die Entscheidung über die Verwendung der Mittel trifft der Inhaber des Grants allein. Der Grant sollte über drei Jahre laufen und pro Jahr mit etwa DM 10.000 ausgestattet sein. Einziges Kriterium für die Entscheidung über einen solchen Grant sollte die bisherige Leistung sein. Der Topf sollte so groß sein, dass alle aktiven Leute eine Chance haben. Natürlich stellt sich das Problem, dass junge Leute bei dem Kriterium „bisherige Leistung“ benachteiligt sind. Hier ist zu betonen, dass es nicht um den Umfang der Leistung geht, sondern allein um die Qualität, bei deren Feststellung das Alter angemessen berücksichtigt werden sollte, was dazu führt, dass ein frisch habilitierter Kollege dank seiner hervorragenden Habilitationsschrift einen etablierten Kollegen, der zwar viel, aber nichts Herausragendes in den letzten Jahren publiziert hat, ausstechen kann und sollte.

Ich peile mal über den Daumen, dass im Schnitt pro Fachbereich vier Kollegen so stark sind, dass sie einen Grant erhalten sollten. Das macht bei geschätzt 70 Fachbereichen etwa 300 Grants. Wenn man von 10.000 DM pro Jahr ausgeht, sind das 3 Mio. DM, das ist gerade mal der Betrag von einem SFB und einer Forschergruppe.

Als nächste Stufe würde ich einen zentralen Gästetopf einrichten, aus dem längerfristige Einladungen 
von einem Monat bis zu einem Jahr finanziert werden können. Hier sollte jeder antragsberechtigt sein, egal ob er schon einen Grant hat oder nicht. Begründung für einen solchen Antrag könnte

(a) eine gemeinsame Arbeit sein, die durch Vorarbeiten schon eine gewisse Gestalt angenommen hat;

(b) der Wunsch sein, dass der Gast eine Vorlesung oder Vortragsreihe zu einem besonders aktuellen Forschungsthema hält;

(c) der Wunsch sein, einen jungen herausragenden PostDoc anzuziehen, der einerseits von den Forschungsaktivitäten des Einladenden besonders profitieren könnte und andererseits wichtige Farbtupfer in der vorhandenen Forschungslandschaft setzen könnte.

Je nach Entwicklungsstand der Besucher würde ein solcher Aufenthalt zwischen 100.000 und 150.000 DM kosten, wobei der Betrag bei Kollegen, die ein Sabbatical haben, viel geringer sein könnte.

Wie groß dieser Topf sein sollte, ist schwer einzuschätzen. Wenn man im Durchschnitt für jeden Fachbereich zwei Ganzjahresstellen ansetzt, kommt man auf 10-15 Mio. DM. Ich möchte der Deutlichkeit halber betonen, dass auf keinen Fall mit der Gießkanne verteilt werden sollte. Entscheidende Kriterien sollten frühere Leistungen der Antragsteller und der Kandidaten sein, die eingeladen werden sollen. Wichtig wäre eine möglichst unbürokratische und schnelle Entscheidung über Anträge innerhalb von ein bis zwei Monaten.

Als dritten und letzten Schwerpunkt würde ich ein umfangreiches Doktorandenprogramm einrichten. Dabei würde ich Graduiertenkollegs grundsätzlich beibehalten, allerdings bei der Entscheidung sehr darauf achten, dass

(a) alle Antragsteller sich durch besonders erfolgreiche Forschung ausgezeichnet haben

(b) eine kritische Masse am Ort vorhanden ist, welche ein Kolleg rechtfertigt.

Über die Kollegs (deren Anzahl bei kritischerer Begutachtung etwas kleiner werden könnte) hinaus würde ich einen zentralen Topf mit Doktorandenmitteln einrichten. Dieser sollte so großzügig ausgestattet sein, dass alle starken Doktoranden gefördert werden können. Ich würde pro Fachbereich, der nicht ein Graduiertenkolleg hat, im Schnitt 3 solche Doktorandenstellen vorsehen. Zur Zeit gibt es 23 Graduiertenkollegs. Man müsste also rund 150 solche Doktorandenstellen zusätzlich einrichten. Bei etwa 50.000 DM pro Stipendiat macht das 9 Mio. DM.

Hier möchte ich bemerken, dass die Einrichtung dieser Doktorandenstellen den Markt bei Assistentenstellen entlasten würde, so dass dann jedem dafür geeigneten Postdoc eine Assistentenstelle für 6 Jahre zur Arbeit an einer Habilitation zur Verfügung stehen würde. Das Heisenberg Programm würde ich unbedingt erhalten.

Ich fasse diese Vorschläge in Tabelle 1 zusammen.

Wenn ich mal von 45 Mio. DM für Mathematik im Rahmen der DFG ausgehe, verbleiben noch 8 Mio. für andere Aktivitäten, z. B. Tagungen, Sachmittel, etc.

Es ist klar, worin die Konsequenzen dieses Planes bestünden:

(a) Die mathematischen Sonderforschungsbereiche werden abgeschafft, die zur Zeit allein rund 16 Millionen DM in Anspruch nehmen. Da diese SFBs an Universitäten eingerichtet sind, wo besonders starke Forscher konzentriert sind, wäre die Beteiligung an den obigen Programmen sicher überdurchschnittlich, aber deutlich kleiner als bisher. Mir scheint das auch aus der Sicht zahlreicher Begutachtungen gerechtfertigt.

(b) Die Forschergruppen mit zur Zeit 4 Mio. DM werden abgeschafft. Für die Auswirkungen ähnliches wie unter (a).

(c) Ich würde das Schwerpunktprogramm einsparen, weil dessen Haupteffekt, ein besonders starkes Gebiet national zu fördern, automatisch erreicht würde.

(d) Ich würde die Mittel des Hess- und NoetherProgrammes in das Doktoranden- bzw. Gästeprogramm stecken, ebenso die Mittel der mathematischen Leibniz-Preise. Das könnte weitere 2 Mio. DM einbringen.

Da die Graduiertenkollegs bereits jetzt finanziert werden, beläuft sich der bei meinem Plan benötigte Betrag auf 27 Mio. DM, die oben eingesparten Mittel ergeben 27 Mio. DM. Die Finanzierung wäre also gesichert.

Ich habe bewusst darauf verzichtet, die Höhe der Aufwendungen für das Fach Mathematik zu kritisieren. Jedes Fach sagt, dass es noch mehr braucht und hat dafür vielleicht auch gute Gründe. Mit geht es nur um die Frage, ob man die Mathematik mit den vorhandenen Mitteln besser fördern kann. Davon bin ich, wie oben dargelegt, fest überzeugt.

\section{Abschließende Kommentare}

Der Hauptunterschied meiner Vorstellungen gegenüber der jetzigen Situation besteht in der Abschaffung der Sonderforschungsbereiche und Forschergruppen. Hier werden die Verteidiger des Bestehenden auf die positiven Effekte von Forschungskonzentration verweisen. Ich stimme grundsätzlich $\mathrm{zu}$, dass eine solche Konzentration sich positiv auswirken kann. Sie wird sich aber bei meinem Plan auch 
Tabelle 1: Vorschläge zur Forschungsförderung

\begin{tabular}{lrrr}
\hline Programm & Ausstattung & Anzahl & Finanzvol./DM \\
\hline Grantprogramm & 10000 & 300 & 3000000 \\
Gästeprogramm & $20000-50000$ & 150 & 15000000 \\
Graduiertenkollegs & 500000 & 20 & 10000000 \\
Doktorandenprogramm & 60000 & 150 & 9000000 \\
Gesamtpaket & & & 37000000 \\
\hline
\end{tabular}

ergeben, weil die starken Orte bei den Programmen viel erfolgreicher als schwache sein werden, allerdings nicht so konzentriert und viel abgestufter, d.h. es wird kleine Zentren und mittlere Zentren geben, je nach der Stärke der vor Ort vorhandenen Kollegen.

Ich würde die Sonderforschungsbereiche und Forschergruppen übrigens verteidigen, wenn wir nicht neben den kleinen und mittleren Zentren zwei große Forschungsinstitute mit breiter fachlicher Ausrichtung hätten, nämlich die beiden Max-PlanckInstitute. Außerdem gibt es noch die anderen oben erwähnten wie Weierstraß-Institut, Zuse-Institut und das Institut für diskrete Mathematik in Bonn. Insbesondere die beiden Max-Planck-Institute ergänzen die Instrumente meines Planes optimal. Ich habe den Eindruck, dass auf diese Weise ein viel abgerundeteres Gebilde entstehen würde.

Zum Abschluss möchte ich noch ein Wort in eigener Sache als Direktor des Oberwolfacher Institutes sagen. Mir scheint das Institut mit seinen beiden Hauptaktivitäten, den wöchentlichen Workshops und dem Research-in-Pairs-Programm eine sehr gute Ergänzung zu dem Skizzierten zu sein. Hier wird das Bedürfnis nach wissenschaftlicher Kommunikation und Gemeinschaftsforschung breit und aktuell auf hohem Niveau befriedigt. Leider sind in den letzten Jahren die finanziellen Schwierigkeiten in Oberwolfach enorm geworden. Angesichts von 45 Mio. DFG-Mitteln und schätzungsweise 15 Mio. für die anderen Institute sollte der benötigte Etat von Oberwolfach, etwa 3 Mio (also $5 \%$ dieses Betrages) aufzubringen sein. Dass dies nicht so ist, scheint mir einer der wichtigsten Kritikpunkte an der Forschungsförderung der Mathematik in Deutschland zu sein.
Adresse des Autors
Dr. Matthias Kreck
Fakultät für Mathematik und Informatik
Im Neuenheimer Feld 288
69120 Heidelberg
kreck@mathi . uni-heidelberg.de

\section{mathemas ordinate www.ordinate.de}

× 0431-2374500/凶-3288812,info@ordinate.de $\rightarrow$ Software for mathematical people!

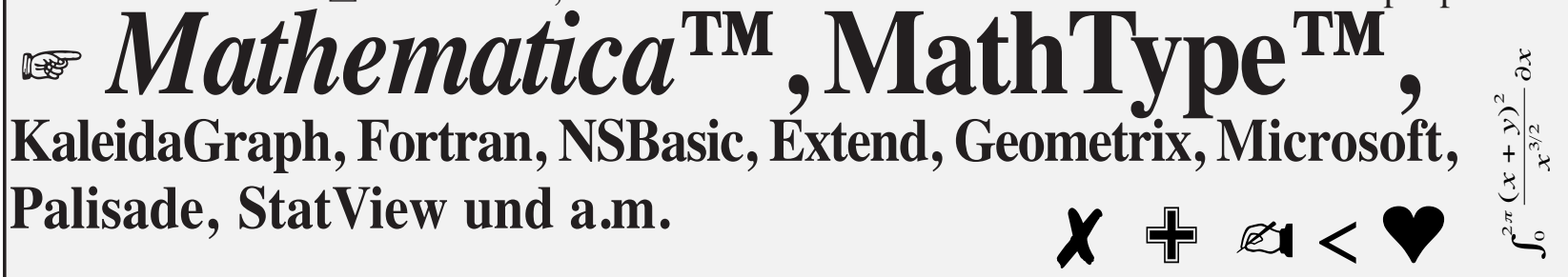

mathemas ordinate, Dipl. Math.Carsten Herrmann, M. Sc. Königsbergerstr. 97, 24161 Altenholz

Fast 20 Jahre Erfahrung mit Software-Distribution! 